Article type: Full Paper

\title{
Triphenylamine Photoconductive Polymers for High Performance Photorefractive Devices
}

Ha Ngoc Giang, Kenji Kinashi, Wataru Sakai, Naoto Tsutsumi*

Keywords: composite, photoconductor, photorefractive, polymer, triphenylamine.

H. N. Giang, Prof. K. Kinashi, Prof. W. Sakai, Prof. N.Tsutsumi

Department of Macromolecular Science and Engineering, Kyoto Institute of Technology,

1Hashigami-cho, Matsugasaki, Sakyo, $\quad$ Kyoto 606-8585, Japan

E-mail: tsutsumi@kit.ac.jp 


\section{Abstract}

Photorefractive performances of the composites using two kinds of photoconductive triphenylamine-based polymer have been compared and investigated. One polymer is poly(4(diphenylamino)benzyl acrylate) (PDAA). The other is newly synthesized one of photoconductive acrylate polymer with methoxy substituted triphenylamine pendant, poly(4-((4methoxyphenyl)(phenyl)amino)benzyl acrylate) (PMPAA). The methoxy substituent in PMPAA does not only shift the highest occupied molecular orbital (HOMO) level of the polymer, but also effectively enhances the chromophore orientation. Larger phase shift is confirmed by using the modified photoconductive polymer of PMPAA. The plasticizer of (4(diphenylamino)phenyl)methanol $(\mathrm{TPAOH})(\mathrm{IP}=-5.64 \mathrm{eV})$ works as an effective trap in the PDAA $(\mathrm{IP}=-5.69 \mathrm{eV})-$ based composite, resulting in higher diffraction efficiency. Diffraction efficiency of $70 \%$ and fast response time of $25 \mathrm{~ms}$ (dominant) is measured at $532 \mathrm{~nm}$ under the moderate electric field of $45 \mathrm{~V} / \mu \mathrm{m}$. 


\section{Introduction}

Photorefractive (PR) effect has received much attention because of its updatable holographic property. PR materials can be applied to varied applications include holography, data storage, optical phase conjugation, image amplification, novelty filtering and edge enhancement.[1, 2] PR polymers and composites have been considered as an alternative to the inorganic PR crystals due to the benefit from their organic properties such as low cost, ease in processability, etc. A great development in material design, PR performances and applications has been reported.[3-6] Typical components for PR effect include a photoconductive polymer, a nonlinear optical (NLO) chromophore, a plasticizer and a sensitizer. The photoconductive polymer plays a main role to provide a charge transport medium. Poly(vinyl carbazole) (PVK) has been successfully applied as a polymer matrix for PR composite.[7] However, PVK has some drawbacks such as low hole mobility and high glass transition temperature $\left(T_{g}\right)$ of $>200{ }^{\circ} \mathrm{C}$. The low hole mobility limits speed of space-charge field formation and consequently leads to slow PR response time. A photoconductive polymer with higher level of the highest occupied molecular orbital (HOMO), i.e more readily ionized, is preferred to obtain an appropriate photoconductivity. In addition, the use of the high HOMO level polymer for PR composites could avoid the possibility of accumulation of trap caused by a chromophore with HOMO level which lies above the charge transport manifold.[8] In the case of a high $T_{g}$ polymer such as PVK, a plasticizer is added to the composite to decrease the $T_{g}$ and enhance the chromophore re-orientation process which plays as an essential factor for the high PR performances.[9] To make the composite less inert, the photoconductive plasticizer ethyl carbazole (ECZ) is widely used with PVK and even with bistriarylamine polymer.[10-16] 
Recently, photoconductive polymers based on triphenylamine have been utilized for fast response PR application because of their fast hole mobility. A vinyl type of triphenylamine polymer, poly((4-diphenylamino)styrene) (PDAS), for PR composite has been reported.[17] However, high intensity of writing beams (up to $3 \mathrm{~W} / \mathrm{cm}^{2}$ ) was applied for the PDAS-based PR composite. The polymer of poly(4-(diphenylamino)benzyl acrylate) (PDAA) with acrylate structure following by a relatively low $T_{g}$ of $75{ }^{\circ} \mathrm{C}$ has been applied to PR composites successfully with a moderate intensity of writing beams.[18] To further improvement and investigation of the PR performances, the side-chain type triphenylamine PDAA is modified with a methoxy group at para-position. In this study, we report a novel photoconductive polymer of poly(4-((4-methoxyphenyl)(phenyl)amino)benzyl acrylate) (PMPAA) and PR performances of the PMPAA-based composite in comparison of the PDAA-based composite. Chromophore orientation and trap density in both composites shall be discussed. 


\section{Experimental Section}

\subsection{Synthesis}

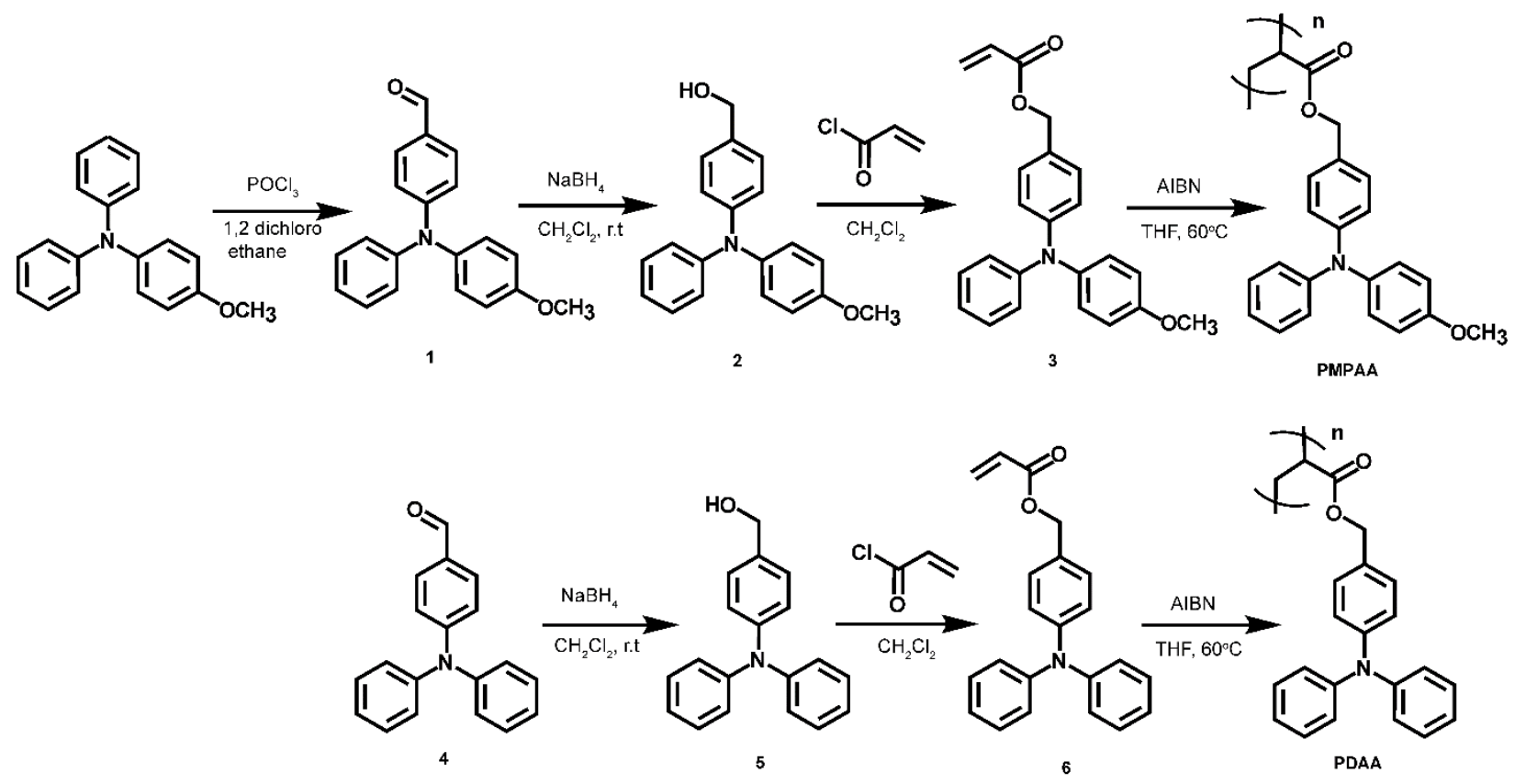

Scheme 1. Synthetic procedure of PMPAA and PDAA.

The synthetic procedure for PDAA and PMPAA is summarized in Scheme 1. All synthesized products were characterized by means of mass spectroscopy (MS) (MicroTOF, BrukerDaltonics Co.), ${ }^{1} \mathrm{H}$ NMR spectroscopy (AV-300, BrukerBioSpin Co., $300 \mathrm{MHz}$, tetramethylsilane -TMSas an internal standard). Weight-average molecular weight $(\mathrm{Mw})$ and number average molecular weight (Mn) were determined by a gel permeation chromatography (GPC) using a Shodex GPC KF-805 + KF-803 column (Showa Denko K. K) and tetrahydrofuran (THF) as an eluent. The molecular weight of the product was calibrated by polystyrene standards. Glass transition temperature $\left(T_{g}\right)$ was determined using a DSC 2920 (TA Instruments Co.) at a heating rate of 10 ${ }^{\circ} \mathrm{C} \min ^{-1}$. 
4-((4-Methoxyphenyl)(phenyl)amino)benzaldehyde (1)

$\mathrm{POCl}_{3}(6 \mathrm{~mL}, 64.17 \mathrm{mmol})$ was carefully added to a solution of 4-methoxy-N,N-diphenylaniline (10 g, $36.32 \mathrm{mmol})$, DMF (5 mL, $64.85 \mathrm{mmol})$ and $50 \mathrm{~mL}$ of 1,2- dichloroethane. The mixture was refluxed for $4 \mathrm{~h}$, then cooled to room temperature (r.t), and poured into a saturated aqueous sodium acetate solution $(100 \mathrm{~mL})$. The product was extracted with dichloromethane. The organic layer was dried over anhydrous $\mathrm{MgSO}_{4}$. The solvent was evaporated by a rotary evaporator. The residue was purified by silica gel column chromatography using ethyl acetate: $n$-hexane (2:3) to give (1) (7.6 g, Yield: 69\%). TOF/MS (ESI): found $326.1\left(\mathrm{M}+\mathrm{Na}^{+}\right) .{ }^{1} \mathrm{H}$ NMR $(300 \mathrm{MHz}$, $\left.\mathrm{CDCl}_{3}, \delta\right): 9.79$ (s, 1H, $\left.\mathrm{ArC} \underline{\mathrm{HO}}\right), 7.68-6.89$ (m, 14H, $\left.\mathrm{Ar} \underline{\mathrm{H}}\right), 3.83$ (s, $\left.3 \mathrm{H}, \mathrm{ArOC}_{\underline{3}}\right)$.

(4-((4-Methoxyphenyl(phenyl)amino)phenyl)methanol (2) and (4-(Diphenylamino)phenyl) -methanol (TPAOH) (5)

Aldehyde ((1) or (4)) was added to a solution of $\mathrm{NaBH}_{4}(1$ eq.) in dichloromethane $(20 \mathrm{~mL})$ and methanol $(5 \mathrm{~mL})$. The mixture was stirring for $2 \mathrm{~h}$ at r.t and water $(25 \mathrm{~mL})$ was then added and stirred continuously for $24 \mathrm{~h}$. The mixture was concentrated in vacuo and saturated aqueous $\mathrm{NH}_{4} \mathrm{Cl}$ solution $(30 \mathrm{~mL})$ was then added. The obtained mixture was extracted with dichloromethane, and the resulting organic layer was dried over anhydrous $\mathrm{MgSO}_{4}$ and the solvent was removed totally by a rotary evaporator to give the relative alcohol.

(4-((4-Methoxyphenyl)(phenyl)amino)phenyl)methanol (2). Yield: 90\%. TOF/MS (ESI) $\mathrm{m} / \mathrm{z}$ : found $298.09\left(\mathrm{M}+\mathrm{Na}^{+}\right) .{ }^{1} \mathrm{H} \mathrm{NMR}\left(300 \mathrm{MHz}, \mathrm{CDCl}_{3}, \delta\right): 7.32-6.82(\mathrm{~m}, 14 \mathrm{H}, \mathrm{Ar} \underline{\mathrm{H}}), 4.55(\mathrm{~d}, 2 \mathrm{H}$, $\left.-\underline{\mathrm{C}}_{2}-\mathrm{OH}\right), 3.8\left(\mathrm{~s}, 3 \mathrm{H}, \mathrm{ArOC}_{\underline{H}}\right), 1.59\left(\mathrm{~s}, 1 \mathrm{H},-\mathrm{CH}_{2} \mathrm{O} \underline{\mathrm{H}}\right)$. 
(4-(Diphenylamino)phenyl)methanol (5). Yield: 95\%. TOF/MS (ESI) $\mathrm{m} / \mathrm{z}$ : found 298.09 (M + $\left.\mathrm{Na}^{+}\right) .{ }^{1} \mathrm{H}$ NMR (300 MHz, $\left.\mathrm{CDCl}_{3}, \delta\right): 7.27-6.94(\mathrm{~m}, 14 \mathrm{H}, \mathrm{Ar} \underline{\mathrm{H}}), 4.55\left(\mathrm{~d}, 2 \mathrm{H},-\underline{\mathrm{C}}_{2}-\mathrm{OH}\right), 1.84(\mathrm{~s}$, $\left.1 \mathrm{H},-\mathrm{CH}_{2} \mathrm{O} \underline{\mathrm{H}}\right)$.

4-((4-Methoxyphenyl)(phenyl)amino)benzyl acrylate (3) and 4-(diphenylamino)benzyl acrylate (6)

A solution of (2) or (5), triethylamine (2 eq.) and a catalytic amount of dimethylaminopyridine (DMAP) in dichloromethane $(50 \mathrm{~mL})$ was cooled to $0{ }^{\circ} \mathrm{C}$ by an ice bath. To the mixture, acryloyl chloride (1.5 eq.) was added slowly. The mixture was warmed up to r.t and left stirring for $24 \mathrm{~h}$. Water $(100 \mathrm{~mL})$ was then added to the mixture. The resulting solution was extracted with dichloromethane. The mixture was washed with saturated aqueous $\mathrm{NaHCO}_{3}$, then with water. The organic layer was dried over anhydrous $\mathrm{MgSO}_{4}$ and the solvent was removed by a rotary evaporator. The crude product was purified using silica gel column chromatography using ethylacetate:hexane (3:1) to give acrylate monomer.

4-((4-Methoxyphenyl)(phenyl)amino)benzyl acrylate (3): Yield: 50\%. ${ }^{1} \mathrm{H}$ NMR $(300 \mathrm{MHz}$, $\left.\mathrm{CDCl}_{3}, \delta\right): 6.81-7.25(\mathrm{~m}, 14 \mathrm{H}, \mathrm{Ar} \underline{\mathrm{H}}), 6.44\left(\mathrm{~d}, 1 \mathrm{H}, \mathrm{CH}_{\mathrm{cis}} \underline{\mathrm{H}}_{\text {trans }}=\mathrm{CH}-\mathrm{COO}\right), 6.16\left(\mathrm{q}, 1 \mathrm{H}, \mathrm{CH}_{2}=\mathrm{C} \underline{\mathrm{H}}-\right.$ $\mathrm{COO}), 5.84\left(\mathrm{~d}, 1 \mathrm{H}, \underline{\mathrm{C}}_{\mathrm{cis}} \mathrm{H}_{\text {trans }}=\mathrm{CH}-\mathrm{COO}\right), 5.12\left(\mathrm{~s}, 2 \mathrm{H}, \mathrm{COO}-\underline{\mathrm{C}}_{2}-\mathrm{Ar}\right), 3.8\left(\mathrm{~s}, 3 \mathrm{H}, \mathrm{ArOC} \underline{H}_{3}\right)$.

4-(Diphenylamino)benzyl acrylate (6):Yield: 69\%. ${ }^{1} \mathrm{H}$ NMR (300 MHz, $\left.\mathrm{CDCl}_{3}, \delta\right)$ : 6.9-7.2 (m, $14 \mathrm{H}, \operatorname{Ar} \underline{\mathrm{H}}), 6.45\left(\mathrm{~d}, 1 \mathrm{H}, \mathrm{CH}_{\mathrm{cis}} \underline{\mathrm{H}}_{\text {trans }}=\mathrm{CH}-\mathrm{COO}\right), 6.16\left(\mathrm{q}, 1 \mathrm{H}, \mathrm{CH}_{2}=\mathrm{C} \underline{\mathrm{H}}-\mathrm{COO}\right), 5.84(\mathrm{~d}, 1 \mathrm{H}$, $\left.\mathrm{C}_{\mathrm{C}} \mathrm{H}_{\text {trans }}=\mathrm{CH}-\mathrm{COO}\right), 5.1$ (s, 2H, COO- $\left.\underline{\mathrm{H}}_{2}-\mathrm{Ar}\right)$.

Polymerization of poly(4-(diphenylamino)benzyl acrylate) (PDAA) and poly(4-((4methoxyphenyl)(phenyl)amino)benzyl acrylate) (PMPAA) 
Polymers were synthesized by radical polymerization. Mixture of monomer, AIBN $(0.1 \%)$ and THF was degassed carefully by freeze-pump-thaw method. The mixture was heated up to $60{ }^{\circ} \mathrm{C}$

for $24 \mathrm{~h}$. After reaction, the obtained solution was diluted with THF $(10 \mathrm{~mL})$ and precipitated into $\mathrm{MeOH}(80 \mathrm{~mL})$. The obtained polymer powder was dried in vacuo for $24 \mathrm{~h}$ to give white polymer powder of PDAA (or PMPAA).

PDAA: monomer/THF (2.3 g/ $2 \mathrm{~mL}$ ); Yield: $95 \% ; M_{w}=23,000$ (degree of polymerization (DP) =56); $M_{w} / M_{n}=1.25 ; T_{g}=75^{\circ} \mathrm{C}$.

PMPAA: monomer/THF (2.4 g/ $3.5 \mathrm{~mL})$; Yield: $96 \% ; M_{w}=21,000(\mathrm{DP}=48) ; M_{w} / M_{n}=1.23$; $T_{g}=74{ }^{\circ} \mathrm{C}$.

\subsection{Photorefractive characterization}

PR sample preparation
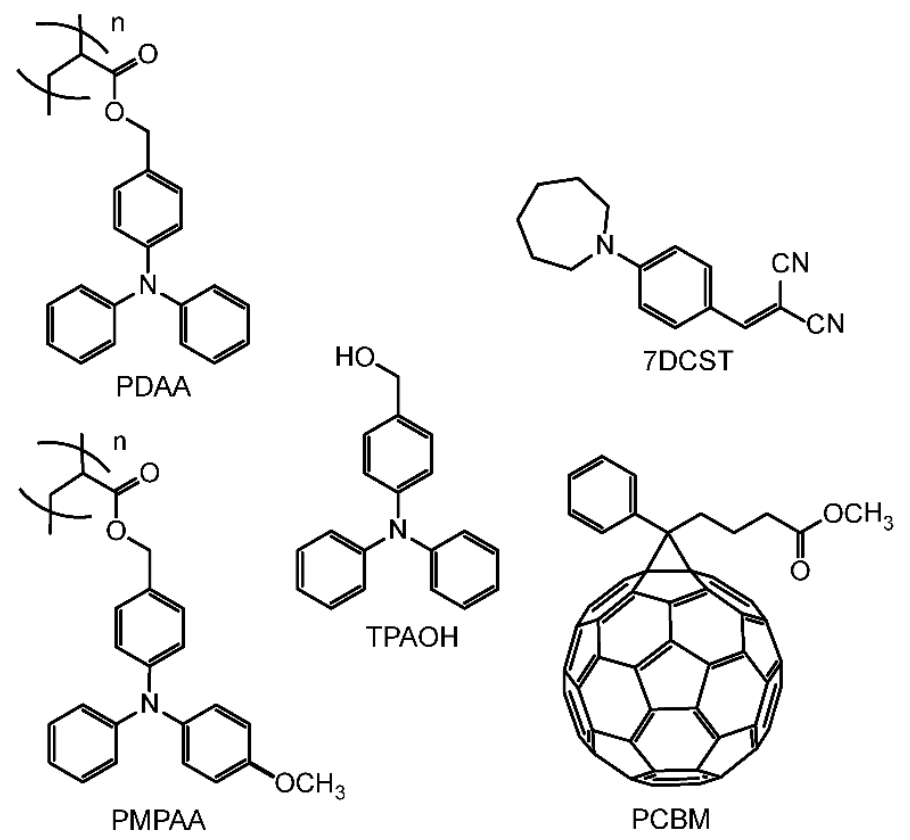

Figure 1. Chemical structures of photoconductive matrix (PDAA or PMPAA), NLO chromophore (7DCST), plasticizer (TPAOH), sensitizer (PCBM). 
The polymer of PDAA or PMPAA was mixed with NLO chromophore 2-(4-(azepan-1yl)benzylidene)malononitrile (7DCST), plasticizer (4-(diphenylamino)phenyl)methanol (TPAOH) and sensitizer phenyl-C61-butyric acid methyl ester (PCBM) in THF. The chemical structural formulae and abbreviations of the components in the PR composite are shown in Figure 1. The concentration of 7DCST was fixed at $30 \mathrm{wt} \%$ in all samples while the concentration of the photoconductive matrix (PDAA or PMPAA) and the plasticizer (TPAOH) were varied. The mixture was left stirring overnight. Then, the mixture was cast on a hot plate at $70{ }^{\circ} \mathrm{C}$ for $24 \mathrm{~h}$ and followed by drying in vacuum oven at $60{ }^{\circ} \mathrm{C}$ for $24 \mathrm{~h}$. The obtained composite was sandwiched between two indium-tin-oxide (ITO) coated glasses as the composite was melted at $150{ }^{\circ} \mathrm{C}$. Thickness of the composite film was controlled by using Teflon spacers.

Degenerate Four-Wave Mixing (DFWM)

Diffraction efficiency of the PR sample was measured using a degenerate four-wave mixing (DFWM) technique. The holographic gratings were written in the sample by two $s$-polarized beams of He-Ne laser (at $633 \mathrm{~nm}, 10 \mathrm{~mW}$, Melles-Griot, USA, $250 \mathrm{~mW} / \mathrm{cm}^{2}$ ) or DPSS laser $\left(\mathrm{Samba}^{\mathrm{TM}}, 532 \mathrm{~nm}, 25 \mathrm{~mW}\right.$, Cobolt, Sweden, $\left.650 \mathrm{~mW} / \mathrm{cm}^{2}\right)$ intersected in the sample at the incidence angles of $40^{\circ}$ and $55^{\circ}$. A weak intensity $p$-polarized reading (probe) beam which counter-propagated to one of the writing beams was diffracted by the refractive index grating in the sample film. The diffracted signal was detected by a photodiode detector. The probe beam that transmitted through the sample film was also detected by another photodiode detector. From the intensity of the transmitted beam $\left(I_{t}\right)$ and the diffracted beam $\left(I_{d}\right)$, the diffraction efficiency ( $\eta$ ) was calculated with equation (1):

$\eta \%=\frac{I_{d}}{I_{t}+I_{d}} \times 100$ 
The PR response time for the composite was measured using the same geometry. The diffraction efficiency growth was fitted with bi-exponential function equation (2):

$$
\eta=\eta_{0}\left[1-m \exp \left(-t / \tau_{1}\right)-(1-\mathrm{m}) \exp \left(-\mathrm{t} / \tau_{2}\right)\right]
$$

where $\tau_{1}, \tau_{2}$ are time constants with weighing factor of $\mathrm{m}(0 \leq \mathrm{m} \leq 1)$ and $(1-\mathrm{m})$, respectively.

\section{Two-beam coupling measurement (TBC)}

PR optical gain values of the sample were measured using a two-beam coupling (TBC) technique. The same geometric configuration to DFWM technique in which two equal intensity $p$-polarized beams that crossed at the thin film sample was used, except without a probe beam. The intensities of two beams that transmitted through the sample film were detected using photodiode detectors to evaluate the optical gain using equation (3):

$$
\Gamma=\frac{1}{d}\left(\cos \theta_{A} \cdot \ln \frac{I_{A}\left(I_{B} \neq 0\right)}{I_{A}\left(I_{B}=0\right)}-\cos \theta_{B} \cdot \ln \frac{I_{B}\left(I_{A} \neq 0\right)}{I_{B}\left(I_{A}=0\right)}\right)
$$

Where $d$ is the thickness of the sample film; $\theta_{A}, \theta_{B}$ are the internal angles of two beams with respect to the normal; $I_{A}$ and $I_{B}$ are the intensities of two beams after the sample film.

\subsection{Characterization of electro-optic response of the composite}

A Mach-Zehnder (MZ) interferometer was employed using a modulated poling field to observe the electro-optic response of the PR composite. The PR composite was placed in one arm of the interferometer with an applied voltage device. A laser at $830 \mathrm{~nm}$ is used in $\mathrm{MZ}$ interferometer because most of the investigated PR composites have small or neglected absorption at this wavelength. A constant poling field of $40 \mathrm{~V} / \mu \mathrm{m}$ was applied, and a stepwise modulating voltage with frequency of $1 \mathrm{~Hz}$ and amplitude of $\pm 4 \mathrm{~V} / \mu \mathrm{m}$ was superposed onto this. 


\subsection{Photocurrent measurement}

A typical experimental run for photocurrent measurement included the following: the electric

field $(40 \mathrm{~V} / \mu \mathrm{m})$ was turned on for $10 \mathrm{~min}$ in dark condition. Then, a laser at $640 \mathrm{~nm}(900$ $\mathrm{mW} / \mathrm{cm}^{2}$ ) as an exciting source was turned on, the current was monitored in $10 \mathrm{~s}$. 


\section{Results and Discussion}

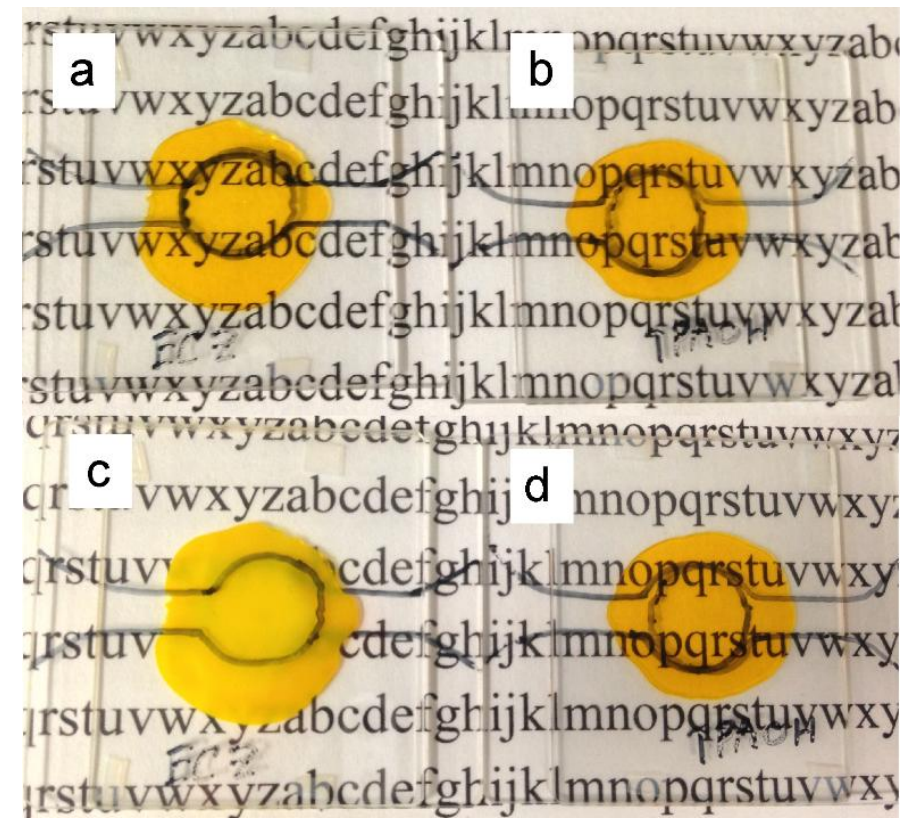

Figure 2. Photorefractive composites with using different plasticizers. (a) PDAA/7DCST/ECZ (45/30/25)-right after preparation; (b) PDAA/7DCST/TPAOH (45/30/25)-right after preparation; (c) PDAA/7DCST/ECZ (45/30/25) after 24 h; (d) PDAA/7DCST/TPAOH (45/30/25)-after 24 h.

PDAA and PMPAA were synthesized with a comparable molecular weight, degree of polymerization and polydispersity (see 2 . Experimental section). Because of that, the dependence of molecular weight on PR effect can be neglected when PR properties of the two kinds of composite were compared. Due to a similar polymer backbone structure of acrylate type, both polymers PDAA and PMPAA possess very close $T_{\mathrm{g}}$ values of $75{ }^{\circ} \mathrm{C}$ and $74{ }^{\circ} \mathrm{C}$, respectively. The ionization potential (IP) values of PDAA and PMPAA are $-5.69 \mathrm{eV}$ and $-5.57 \mathrm{eV}$, respectively. A modification by a strong electron donating group $\left(-\mathrm{OCH}_{3}\right)$ at para-position has slightly shifted the HOMO level of the photoconductive polymer. Instead of the popular plasticizer ethyl carbazole (ECZ), the new plasticizer of TPAOH shown in Figure 1 has been applied to the PR composite. The main purpose of the TPAOH plasticizer is to increase the concentration of the 
photoconductive triphenylamine in the composite which can assist to have a better photoconductivity. In addition, the use of TPAOH has been found to improve the shelf life time of the PR composite. Two samples: PDAA/7DCST/ECZ and PDAA/7DCST/TPAOH with a weight ratio of 45/30/25 have been prepared. Figure 2 shows the picture of PR samples right after preparation and after $24 \mathrm{~h}$ storage at room temperature. As can be seen, the sample with ECZ showed a severe re-crystallization of NLO chromophore and a totally non-transparent sample was observed after $24 \mathrm{~h}$. In some cases, in the sample with ECZ, the sign of recrystallization appeared only after a few hours. While in the sample with TPAOH, the transparency was maintained for not only $24 \mathrm{~h}$ but also one month. All of the PR samples which were used in the present study showed no sign of degradation caused by the chromophore recrystallization during the investigation time.
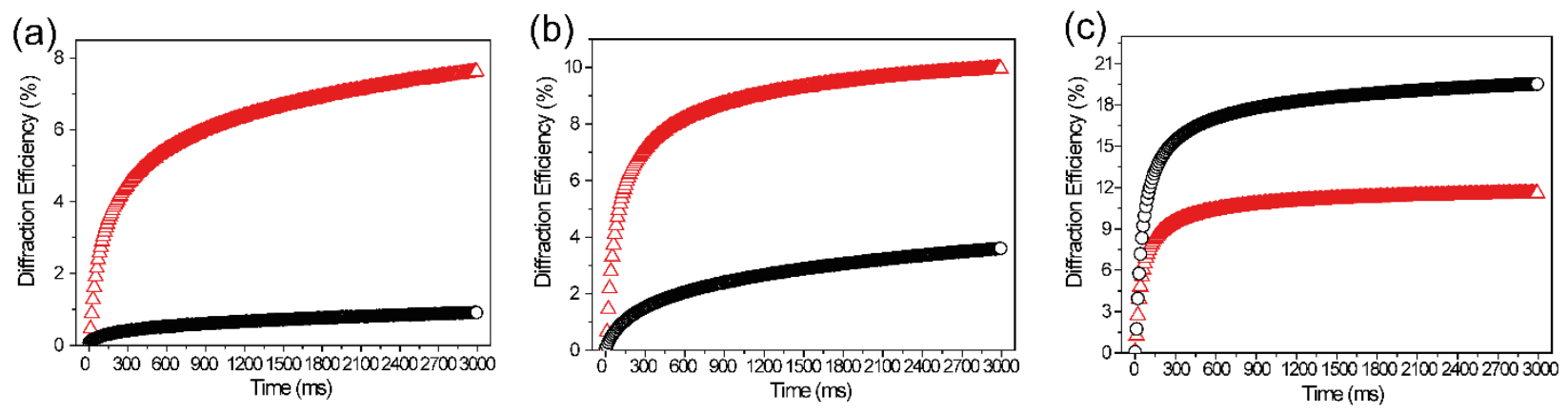

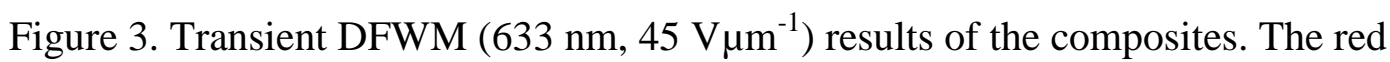
triangle: PMPAA-based composites; the black circle: PDAA-based composites. Polymer (PDAA or PMPAA)/7DCST/TPAOH/PCBM: (a) 55/30/14/1, (b) 50/30/19/1, (c) 45/30/24/1. 
Table 1. Photorefractive parameters of the PDAA-based composites

\begin{tabular}{|c|c|c|c|c|c|c|c|}
\hline PDAA/7DCST/TPAOH/PCBM & $\begin{array}{c}\text { Thickness } \\
(\mu \mathrm{m})\end{array}$ & $\begin{array}{l}\eta^{(c)} \\
(\%)\end{array}$ & $\begin{array}{c}\text { Response } \\
\text { time }^{(c)}\end{array}$ & $\begin{array}{c}\text { Optical } \\
\text { gain }^{(\mathrm{c})} \\
\Gamma\left(\mathrm{cm}^{-1}\right)\end{array}$ & $\Delta \mathbf{n}$ & $\begin{array}{l}\text { Phase } \\
\text { shift } \\
\Phi\left(^{\circ}\right)\end{array}$ & $\begin{array}{c}\text { Absorption } \\
\text { coefficient } \\
\boldsymbol{\alpha}\left(\mathrm{cm}^{-1}\right)\end{array}$ \\
\hline $55 / 30 / 14 / 1^{(a)}$ & 97.5 & 1 & $\begin{aligned} \tau_{1} & =80 \mathrm{~ms} \\
\tau_{2} & =1834 \mathrm{~ms} \\
\mathrm{~m} & =0.305 \\
\tau & =1299 \mathrm{~ms}\end{aligned}$ & 7 & $1.9 \times 10^{-4}$ & 10.8 & 24 \\
\hline $50 / 30 / 19 / 1^{(a)}$ & 92.5 & 4 & $\begin{aligned} \tau_{1} & =136 \mathrm{~ms} \\
\tau_{2} & =1741 \mathrm{~ms} \\
\mathrm{~m} & =0.303 \\
\tau & =1254 \mathrm{~ms}\end{aligned}$ & 21 & $3.9 \times 10^{-4}$ & 15.4 & 8 \\
\hline $45 / 30 / 24 / 1^{(a)}$ & 87.5 & 19 & $\begin{array}{l}\tau_{1}=64 \mathrm{~ms} \\
\tau_{2}=761 \mathrm{~ms} \\
\mathrm{~m}=0.706 \\
\tau=266 \mathrm{~ms}\end{array}$ & 72 & $9.4 \times 10^{-4}$ & 22.65 & 19 \\
\hline $45 / 30 / 24 / 1^{(b)}$ & 87.5 & 70 & $\begin{aligned} \tau_{1} & =25 \mathrm{~ms} \\
\tau_{2} & =273 \mathrm{~ms} \\
\mathrm{~m} & =0.791 \\
\tau & =77 \mathrm{~ms}\end{aligned}$ & $-n-$ & $1.7 \times 10^{-3}$ & $-n-$ & $-n-$ \\
\hline
\end{tabular}
(a) Writing beams are at $633 \mathrm{~nm}$.
(b) Writing beams are at $532 \mathrm{~nm}$.
(c) Measured at $45 \mathrm{~V} / \mu \mathrm{m}$. $\tau=m \tau_{1}+(1-\mathrm{m}) \tau_{2}$
-n- no data 
Table 2. Photorefractive parameters of the PMPAA-based composites.

\begin{tabular}{|c|c|c|c|c|c|c|c|}
\hline PMPAA/7DCST/TPAOH/PCBM & $\begin{array}{c}\text { Thickness } \\
\text { ( } \mu \mathrm{m})\end{array}$ & $\begin{array}{l}\eta^{(\mathbf{c})} \\
(\%)\end{array}$ & $\begin{array}{c}\text { Response } \\
\text { time }^{(\mathrm{c})}\end{array}$ & $\begin{array}{l}\text { Optical } \\
\text { gain }^{(\mathrm{c})} \\
\Gamma\left(\mathrm{cm}^{-1}\right)\end{array}$ & $\Delta \mathbf{n}$ & $\begin{array}{l}\text { Phase } \\
\text { shift } \\
\Phi\left(^{\circ}\right)\end{array}$ & $\begin{array}{l}\text { Absorption } \\
\text { coefficient } \\
\alpha\left(\mathrm{cm}^{-1}\right)\end{array}$ \\
\hline $55 / 30 / 14 / 1^{(a)}$ & 92.25 & 8 & $\begin{array}{l}\tau_{1}=96 \mathrm{~ms} \\
\tau_{2}=1271 \mathrm{~ms} \\
\mathrm{~m}=0.492 \\
\tau=696 \mathrm{~ms}\end{array}$ & 46 & $5.8 \times 10^{-4}$ & 24.1 & 47 \\
\hline $50 / 30 / 19 / 1^{(a)}$ & 92 & 10 & $\begin{array}{l}\tau_{1}=81 \mathrm{~ms} \\
\tau_{2}=898 \mathrm{~ms} \\
\mathrm{~m}=0.631 \\
\tau=382 \mathrm{~ms}\end{array}$ & 61 & $6.4 \times 10^{-4}$ & 28.7 & 33 \\
\hline $45 / 30 / 24 / 1^{(a)}$ & 87.5 & 11 & $\begin{aligned} \tau_{1} & =55 \mathrm{~ms} \\
\tau_{2} & =647 \mathrm{~ms} \\
\mathrm{~m} & =0.729 \\
\tau & =214 \mathrm{~ms}\end{aligned}$ & 66 & $7.2 \times 10^{-4}$ & 27.4 & 58 \\
\hline $45 / 30 / 24 / 1^{(b)}$ & 87.5 & 38 & $\begin{aligned} \tau_{1} & =35 \mathrm{~ms} \\
\tau_{2} & =457 \mathrm{~ms} \\
\mathrm{~m} & =0.757 \\
\tau & =137 \mathrm{~ms}\end{aligned}$ & $-n-$ & $1.2 \times 10^{-3}$ & $-n-$ & $-n-$ \\
\hline
\end{tabular}

(a) Writing beams are at $633 \mathrm{~nm}$.

(b) Writing beams are at $532 \mathrm{~nm}$.

(c) Measured at $45 \mathrm{~V} / \mu \mathrm{m}$. $\tau=m \tau_{1}+(1-\mathrm{m}) \tau_{2}$

-n- no data 
Table 1 and Table 2 summarize PR properties for the PDAA-based and the PMPAAbased composites, respectively. Diffraction efficiency growth at $45 \mathrm{~V} / \mu \mathrm{m}$ as a function of time with $633 \mathrm{~nm} \mathrm{He}$ : Ne laser writing beams is shown in Figure 3. In the composites with 14 and 24 wt\% of the TPAOH plasticizer, the PMPAA-based composites shows a significant enhancement over the PDAA-based composite. However, this tendency is reversed at the composition with 24 $\mathrm{wt} \% \mathrm{TPAOH}$ as the diffraction efficiency of the PDAA-based composite is two times larger than that of the PMPAA-based composite. The generation of PR grating can be divided into two steps: formation of a spatially non-uniformed space-charge field and a refractive index change under the overall electric field which is a superposition of the space-charge field and the externally applied field.[12, 19] Hence, the steady state of diffraction efficiency in PR materials is determined by two factors: the strength of space-charge field and the magnitude of the refractive index change under an electric field. To explain the DFWM results, both of the two factors have to be considered. The ability of refractive index change under electric field can be estimated among PR samples by comparing MZ interferometer signals.
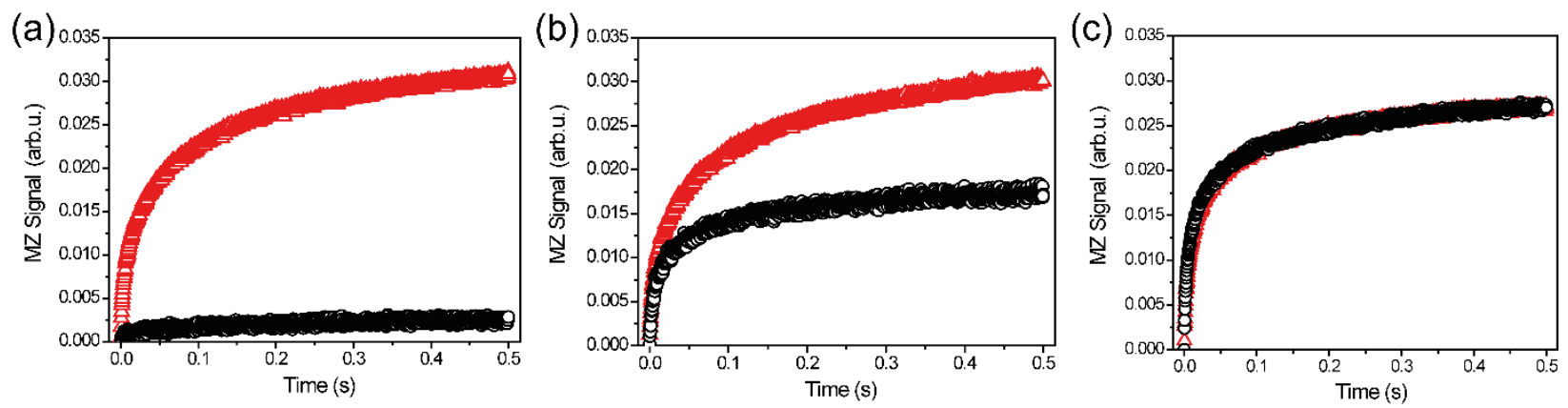

Figure 4. Mach-Zehnder interferometer signals of the composites. The red triangle: PMPAAbased composites; the black circle: PDAA-based composites. Polymer (PDAA or PMPAA)/7DCST/TPAOH/PCBM: (a) 55/30/14/1, (b) 50/30/19/1, (c) 45/30/24/1. 
Figure 4 is the $\mathrm{MZ}$ interferometer results of the relative composites. In MZ interferometer, the signal represents the phase shift variation between two arms of the interferometer. In this case, the MZ signal is directly related to refractive index change in the PR sample under electric field. In a low $T_{g}$ polymeric PR materials, refractive index change under electric field that modulated at a low frequency of $1 \mathrm{~Hz}$ originates from orientational birefringence (BR) and electro-optic (EO) contributions.[9] Both of the contributions depend on the ability of the chromophore to be oriented under the external electric field in the different matrixes as well as the concentration and the type of NLO chromophore itself. In the present case, the same NLO chromophore (7DCST) at the fix concentration (30 wt $\%$ ) was used. It means that the obtained $\mathrm{MZ}$ results under a same electric field strongly reflect the orientation of chromophore. As can be observed in Figure 4, in the composition with 14 and 19 wt $\%$ of plasticizer TPAOH, MZ signals detected in the PMPAA-based composites are significantly larger than those in the PDAA-based composites. The result indicates a dramatic enhancement in chromophore orientation in the PMPAA-based composites. However, in the composition with 24 wt $\%$ of plasticizer, MZ signals of the two kinds of composite are overlapped to each other. The MZ signals in the PDAA-based composite increase with decreasing the PDAA content whereas the MZ signals in the PMPAAbased composite are almost the same irrespective of the PMPAA content. By using 14 wt $\%$ of the plasticizer TPAOH, the chromophore alignment in the PDAA-based composite is seriously restricted at room temperature. An increase in the plasticizer concentration which leads to an decrease in $T_{g}$ improves this situation and with $24 \mathrm{wt} \%$ of TPAOH, the same chromophore alignment ability comparing to the PMPAA-based composites can be reached in the PDAAbased composite. For the PMPAA-based composite, only 14\% plasticizer of TPAOH is enough to have a low $T_{g}$ for chromophore alignment. A good chromophore orientation at a low 
plasticizer concentration is preferred to maximize the photoconductive matrix concentration. In this meaning, PMPAA is a better matrix for the chromophore orientation and PR performances. While the thermal properties of PDAA and PMPAA are similar with a very close $T_{g}$ of 75 and 74 ${ }^{\circ} \mathrm{C}$, respectively (see experimental section), the orientation of the chromophore in the two kinds of composite is very different at 14 and $19 \mathrm{wt} \%$ of TPAOH. The modification in PMPAA from the original polymer PDAA is only an addition of methoxy group $\left(\mathrm{OCH}_{3}\right)$ while the main structure is kept unchanged. There is a possibility that the bulk group $\mathrm{OCH}_{3}$ might provide better environment for chromophore orientation in PMPAA-based composite, resulting in better MZ signals. Following this explanation, modifying PDAA with methoxy group at para-position does not only create a more readily ionized photoconductive polymer with a higher HOMO level but also significantly improve the chromophore orientation in the matrix. In the composition with 14 and $19 \mathrm{wt} \% \mathrm{TPAOH}$, the difference in MZ signals (Figure 4 (a), (b)) is correlated well with the enhancement in diffraction efficiency detected in the PMPAA-based composite. Because of that, the results of DFWM (Figure 3 (a), (b)) can be explained well by the difference in the chromophore orientation in the two kinds of composites. However, with 24 wt $\%$ TPAOH and 45 wt\% polymer concentration, the PDAA-based composites shows larger diffraction efficiency whereas the MZ responses in two kinds of composite are the same. In this case, the MZ interferometer results or chromophore orientation property can no longer explain what happened in the DFWM results. However, an important fact which can be concluded from the MZ measurement is: the ability of changing refractive index under a specific electric field of these composites is the same at this composition. Since the same external electric field of $45 \mathrm{~V} / \mu \mathrm{m}$ was applied for every samples, the difference in diffraction efficiency results can only be explained by the strength of the internal space-charge field. 
(a)

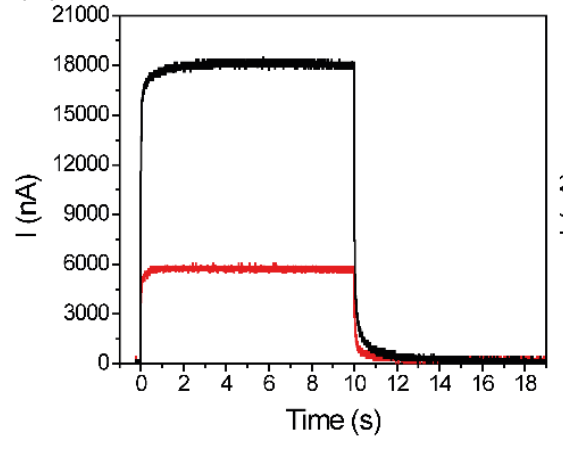

(b)

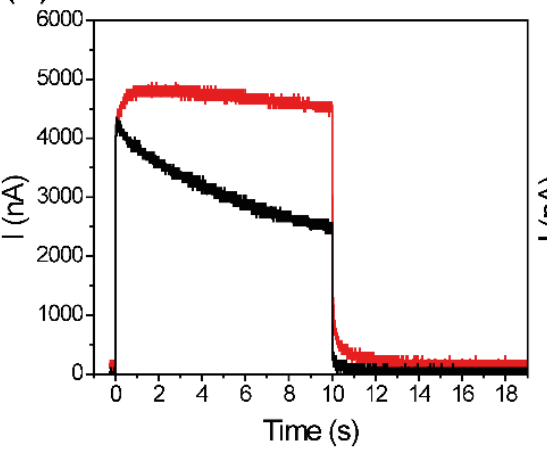

(c)

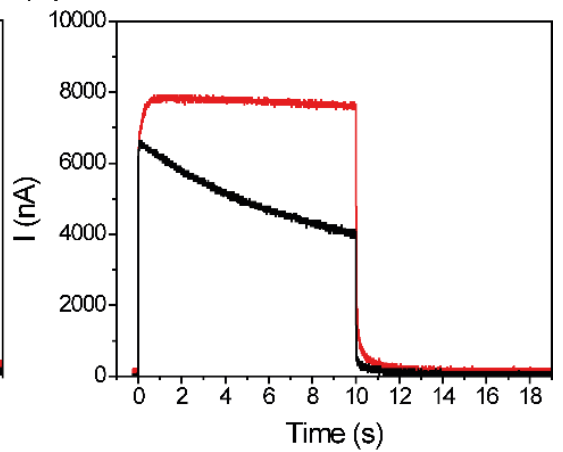

Figure 5. Photocurrent $\left(640 \mathrm{~nm}, 900 \mathrm{~mW} / \mathrm{cm}^{2}, 40 \mathrm{~V} / \mu \mathrm{m}\right)$ results of the composites. The red line:

PMPAA-based composites; the black line: PDAA-based composites. Polymer (PDAA or

PMPAA)/7DCST/TPAOH/PCBM. (a) 55/30/14/1, (b) 50/30/19/1, (c) 45/30/24/1.

The formation of the space-charge field is a complicated process which includes photogeneration of charge carriers, migration of the more mobile charges and trapping process in the dark area.[19, 20] Photocurrent measurement can give a closer look of electronic processes which occur inside the PR samples. Figure 5 shows the photocurrent results for the PDAA and PMPAA-based composites. As can be seen, a significantly large photocurrent comparing to dark current is measured in all composites. Except the samples with 14 wt\% of TPAOH, the PR composites using PMPAA shows larger photocurrent. Improving the photo-generation efficiency by the modification of methoxy $\left(\mathrm{OCH}_{3}\right)$ as an electron donating group at para-position which has increased the HOMO level of the polymer matrix can be used to explain this phenomenon. A similar result of increasing photo-generation efficiency by changing IP values has been reported by Hendrickx et al.[21] However, the largest photocurrent was detected in the PDAA-based composite with the lowest TPAOH ratio and $55 \mathrm{wt} \%$ of polymer. The reason of for this phenomenon has not been made clear. It is worth noting that the MZ signal with this sample is significantly low comparing to others. There is a possibility that with this composition, the PR 
composite is still in a glassy state in which the non-uniform hopping site paths are formed and thus large photocurrent was measured. During $10 \mathrm{~s}$ when the light illuminated the sample, a gradual decay of photocurrent was observed. The filling of deep trap which leads to an increase of recombination center has been attributed to this phenomenon. [20, 22] At 19 and 24 wt\% TPAOH (Figure 5 (b) and (c)), photocurrent curves in the PDAA-based composites show a larger decay than those in the PMPAA-based composites. The result indicates that a larger trap density has been induced in the PDAA-based composite than in the PMPAA-based composite. The values of trap density can be estimated by Kukhtarev model which was described in the previous papers.[10, 23, 24] Table 3 shows the values of the trap-limitted space-charge field $E_{q}$ and number density of traps $N_{t}$.

Table 3. Trap-limitted space-charge field and number density of trap calculated by Kukhtarev model.

\begin{tabular}{|c|c|c|c|c|}
\hline \multirow[b]{2}{*}{ Polymer/7DCST/TPAOH/PCBM } & \multicolumn{2}{|c|}{ PDAA } & \multicolumn{2}{|c|}{ PMPAA } \\
\hline & $\begin{array}{c}\text { Trap-limited } \\
\text { Space-charge } \\
\text { field } E_{q} \\
(\mathrm{~V} / \mu \mathrm{m})\end{array}$ & $\begin{array}{c}\text { Number density } \\
\text { of } \operatorname{trap} N_{T} \\
\left(\mathrm{~cm}^{-3}\right)\end{array}$ & $\begin{array}{c}\text { Trap-limited } \\
\text { Space-charge } \\
\text { field } E_{q} \\
(\mathrm{~V} / \mu \mathrm{m})\end{array}$ & $\begin{array}{c}\text { Number density of } \\
\operatorname{trap} N_{T} \\
\left(\mathrm{~cm}^{-3}\right)\end{array}$ \\
\hline $55 / 30 / 14 / 1$ & 106 & $3.1 \times 10^{16}$ & 45 & $1.3 \times 10^{16}$ \\
\hline $50 / 30 / 19 / 1$ & 73 & $2.1 \times 10^{16}$ & 36 & $1.09 \times 10^{16}$ \\
\hline $45 / 30 / 24 / 1$ & 48 & $1.4 \times 10^{16}$ & 38 & $1.16 \times 10^{16}$ \\
\hline
\end{tabular}

As can be observed, the values of $E_{q}$ and trap density in the PDAA-based composites are larger than those in the PMPAA-based composites. This result is consistent with the result obtained in photocurrent measurement. The difference in trap density of the two kinds of 
composite might relate to the relative position of photoconductive polymer's HOMO level to the others. The IP values of PMPAA, PDAA, 7DCST, PCBM and TPAOH are $-5.57,-5.69,-5.9$, -6.2 and $-5.64 \mathrm{eV}$, respectively. The IP values represent the HOMO level of each component. The PMPAA's HOMO level is higher than that of all the other components whereas the PDAA's HOMO level is lower than that of the plasticizer TPAOH. The slightly higher IP value of TPAOH than PDAA induces the energetically distributed higher potential site which works as a trap site in the charge transport manifold. Namely, a plasticizer TPAOH could act as an effective trap in the PDAA-based composite. It is known that larger trap density leads to larger spacecharge field. By using $24 \mathrm{wt} \% \mathrm{TPAOH}$ and $45 \mathrm{wt} \%$ polymer concentration, the chromophore orientation of the two composites is the same, and thus higher diffraction efficiency in the PDAA-based composites as shown in Figure 3 (c) is ascribed to the larger space-charge field created by the larger amount of trap. Tsujimura et al. has reported a significantly small diffraction efficiency (less than 2\%) with triphenylamine plasticizer due to the low trap density comparing to using ECZ plasticizer.[17] By an optimization of chromophore orientation and trap density by using TPAOH plasticizer, high diffraction efficiency could be detected.

As can be observed in Table 1 and 2, it is clearly shown that diffraction efficiency for the PMPAA-based composites is not changed so much irrespective of the composition of components. Whereas the PDAA-based composites gave rise to the strong dependence of diffraction efficiency on the composition of components. The behavior relates to the good chromophore orientation and the low trap density by using PMPAA as the photoconductive matrix. As a good matrix for the chromophore orientation, a large MZ signal could be detected even at a small amount of 9 wt $\%$ of TPAOH plasticizer (Figure 4a). However, the low trap density which leads to the small space-charge field limits the diffraction efficiency. The growth 
of diffraction efficiency was fitted well with bi-exponential function and the dominant response time with its weigh factor was reported. The fastest response time was detected with the largest plasticizer concentration. Diffraction efficiency and response time with $532 \mathrm{~nm}$ laser as the writing beams for the composition with $24 \mathrm{wt} \% \mathrm{TPAOH}$ and $45 \mathrm{wt} \%$ polymer concentration were also listed in Table 1 and Table 2 . At the same externally applied electric field of $45 \mathrm{~V} / \mu \mathrm{m}$, the diffraction efficiency values in both composites were increased dramatically comparing to the results in which $633 \mathrm{~nm}$ writing beams have been used. The diffraction efficiency originates from changes in refractive index modulation $(\Delta \mathrm{n})$ which can be calculated from Kogelnik's coupled-wave theory. [25] As can be seen in Table 1 and 2, the magnitude of refractive index modulation in which the $532 \mathrm{~nm}$ writing beams were used is larger than that when the $633 \mathrm{~nm}$ writing beams were used. A strong space-charge field caused by improving charge generation with $532 \mathrm{~nm}$ laser has been assigned to explain this phenomenon.[18] The PMPAA-based composite reaches $38 \%$ diffraction efficiency with a fast response time of $35 \mathrm{~ms}$ (dominant, $\mathrm{m}=$ 0.757). Due to larger trap density, the PDAA-based composite shows $70 \%$ diffraction efficiency with a fast time constant of $25 \mathrm{~ms}$ (dominant, $\mathrm{m}=0.791$ ). In comparison to the previous study, $[17,18]$ the results indicate that high diffraction efficiency and fast response time can be obtained in this type of material with a moderate laser intensity and applied field. Although better PR properties were achieved in the PDAA-based composite with the appropriate amount of the plasticizer TPAOH, a careful consideration has to be taken into account. The IP value of TPAOH $(-5.64 \mathrm{eV})$ is higher than PDAA $(-5.69 \mathrm{eV})$. The present component with a little higher HOMO level than charge transport manifold can lead to the accumulation of the compensate traps. Prolonged optical exposure during PR operation can create excessive traps which reduced the photoconductivity and thus suppressed the PR performances. The 
phenomenon is related to the pre-illumination effect which was found in PVK due to its energy level comparing to NLO chromophore.[19, 20]

One of the most important properties of PR material is the asymmetric energy transfer between two beams. The space-charge field formed by a non-uniform illumination induces a refractive index grating. The grating is shifted a spatial angle of $\Phi$ comparing to the optical interference pattern. This nonlocal grating leads to an energy transfer between two beams. The existence of TBC signal and phase shift is a fingerprint for PR effect. $p$-Polarization beams were used as writing beams because it is relatively easier to observe the effect. The asymmetric energy transfer with $p$-polarization beams is significantly larger than that using s-polarization.[26] The direction of applied electric field was chosen to reduce beam fanning. Values of phase shift were calculated by coupled-wave theory from the values of diffraction efficiency and optical gain.[10, $24,25]$ The results of optical gain, absorption coefficient and phase shift values are summarized in Table 1 and 2. The performance of optical gain follows the same tendency to the diffraction efficiency results. Except the composition with $14 \mathrm{wt} \% \mathrm{TPAOH}$, the net gain values $(\Gamma-\alpha)$ which is favorable to utilize the material for TBC applications were achieved. Interestingly, the calculated phase shift values detected in the PMPAA-based composites are larger than that in the PDAA-based composites for all of investigated samples (Table 1 and 2). The difference in trap density between both composites can be applied to explain this phenomenon. A photoconductive matrix with higher HOMO level than the other PR components meets less probability to trap the mobile charges. Thus, the electrical charges, the holes, can migrate a longer distance after free carrier formation from the ion pair. As a result, the larger phase shift was induced by using PMPAA. 


\section{Conclusions}

PR performances of the two kinds of triphenylamine-based composites have been investigated. The chemical modification by a methoxy group at para-position to triphenylamine has not only raised HOMO level but also provided a better environment for molecular alignment and orientation. The chromophore orientation was effectively enhanced by using the modified polymer of PMPAA. Larger phase shift were obtained in the all PMPAA-based composites and net gain was detected in the present composites. Photoconductive plasticizer TPAOH (IP $=-5.64$ $\mathrm{eV})$ worked as an effective trap for the charge transport manifold in the PDAA (IP=-5.69 eV) based composite, which led to larger trap density than that in PMPAA-based composite. High diffraction efficiency of $70 \%$ with fast response time of $25 \mathrm{~ms}$ (dominant) could be detected in PDAA-based composite at the moderate laser intensity and electric field of $45 \mathrm{~V} / \mu \mathrm{m}$. 


\section{Acknowledgements}

Authors thank to Prof. Kawabe, Chitose Inst. Sci. Tech. for measuring IP; Dr. Takafumi Sassa and Dr. Takahashi Fujihara (RIKEN, The Institute of Physical and Chemical Research, Japan) for providing apparatus and technical advices in $\mathrm{MZ}$ interferometer and photocurrent measurement. This work is partly supported by the program for Strategic Promotion of Innovative Research and Development (s-Innovation), Japan Science and Technology Agency (JST). 


\section{References}

[1] P. Gunter, J.-P. Huignard, Photorefractive Materials and Their Applications 2, in: Photorefractive Materials and Their Applications, Springer, 2007.

[2] B. Lynn, P.-A. Blanche, N. Peyghambarian, Photorefractive polymers for holography, J. Polym. Sci. Part B Polym. Phys., 52 (2014) 193-231.

[3] W.E. Moerner, S.M. Silence, Polymeric photorefractive materials, Chem. Rev., 94 (1994) 127-155.

[4] O. Ostroverkhova, W.E. Moerner, Organic Photorefractives: Mechanisms, Materials, and Applications, Chem. Rev., 104 (2004) 3267-3314.

[5] S. Köber, M. Salvador, K. Meerholz, Organic Photorefractive Materials and Applications, Adv. Mater., 23 (2011) 4725-4763.

[6] J. Thomas, C.W. Christenson, P.-A. Blanche, M. Yamamoto, R.A. Norwood, N. Peyghambarian, Photoconducting Polymers for Photorefractive 3D Display Applications $\dagger$, Chem. Mater., 23 (2010) 416-429.

[7] K. Meerholz, B.L. Volodin, Sandalphon, B. Kippelen, N. Peyghambarian, A photorefractive polymer with high optical gain and diffraction efficiency near 100\%, Nature, 371 (1994) 497500.

[8] J.A. Herlocker, C. Fuentes-Hernandez, K.B. Ferrio, E. Hendrickx, P.A. Blanche, N. Peyghambarian, B. Kippelen, Y. Zhang, J.F. Wang, S.R. Marder, Stabilization of the response time in photorefractive polymers, Appl. Phys. Lett., 77 (2000) 2292-2294.

[9] W.E. Moerner, S.M. Silence, F. Hache, G.C. Bjorklund, Orientationally enhanced photorefractive effect in polymers, J. Opt. Soc. Am. B, 11 (1994) 320-330.

[10] N. Tsutsumi, A. Dohi, A. Nonomura, W. Sakai, Enhanced performance of photorefractive poly(N-vinyl carbazole) composites, J. Polym. Sci. Part B Polym. Phys., 49 (2011) 414-420.

[11] B. Kippelen, S.R. Marder, E. Hendrickx, J.L. Maldonado, G. Guillemet, B.L. Volodin, D.D. Steele, Y. Enami, Sandalphon, Y.J. Yao, J.F. Wang, H. Röckel, L. Erskine, N. Peyghambarian, Infrared Photorefractive Polymers and Their Applications for Imaging, Science, 279 (1998) 5457.

[12] R. Bittner, C. Bräuchle, K. Meerholz, Influence of the Glass-Transition Temperature and the Chromophore Content on the Grating Buildup Dynamics of poly(N-vinylcarbazole)-based Photorefractive Polymers, Appl. Opt., 37 (1998) 2843-2851.

[13] J. Thomas, C. Fuentes-Hernandez, M. Yamamoto, K. Cammack, K. Matsumoto, G.A. Walker, S. Barlow, B. Kippelen, G. Meredith, S.R. Marder, N. Peyghambarian, Bistriarylamine Polymer-Based Composites for Photorefractive Applications, Adv. Mater., 16 (2004) 2032-2036. [14] P.-A. Blanche, S. Tay, R. Voorakaranam, P. Saint-Hilaire, C. Christenson, T. Gu, W. Lin, D. Flores, P. Wang, M. Yamamoto, J. Thomas, R.A. Norwood, N. Peyghambarian, An Updatable Holographic Display for 3D Visualization, J. Disp. Technol., 4 (2008) 424-430.

[15] S. Tay, P.A. Blanche, R. Voorakaranam, A.V. Tunc, W. Lin, S. Rokutanda, T. Gu, D. Flores, P. Wang, G. Li, P. St Hilaire, J. Thomas, R.A. Norwood, M. Yamamoto, N. Peyghambarian, An updatable holographic three-dimensional display, Nature, 451 (2008) 694-698.

[16] P.A. Blanche, A. Bablumian, R. Voorakaranam, C. Christenson, W. Lin, T. Gu, D. Flores, P. Wang, W.Y. Hsieh, M. Kathaperumal, B. Rachwal, O. Siddiqui, J. Thomas, R.A. Norwood, M. 
Yamamoto, N. Peyghambarian, Holographic three-dimensional telepresence using large-area photorefractive polymer, Nature, 468 (2010) 80-83.

[17] S. Tsujimura, K. Kinashi, W. Sakai, N. Tsutsumi, High-Speed Photorefractive Response Capability in Triphenylamine Polymer-Based Composites, Appl. Phys. Express, 5 (2012).

[18] H.N. Giang, K. Kinashi, W. Sakai, N. Tsutsumi, Photorefractive response and real-time holographic application of a poly(4-(diphenylamino)benzyl acrylate)-based composite, Polym. J., 46 (2014) 59-66.

[19] O. Ostroverkhova, Organic and Polymeric Photorefractive Materials and Devices, in: S. Sam-Shajing, D. Larry R. (Eds.) Introduction to Organic Electronic and Optoelectronic Materials and Devices, CRC Press, Taylor \& Francis Group, 2008, pp. 607-638.

[20] O. Ostroverkhova, K.D. Singer, Space-charge dynamics in photorefractive polymers, J. Appl. Phys., 92 (2002) 1727-1743.

[21] E. Hendrickx, B. Kippelen, S. Thayumanavan, S.R. Marder, A. Persoons, N. Peyghambarian, High photogeneration efficiency of charge-transfer complexes formed between low ionization potential arylamines and C60, J. Chem. Phys, 112 (2000) 9557-9561.

[22] L. Kulikovsky, D. Neher, E. Mecher, K. Meerholz, H.H. Hörhold, O. Ostroverkhova, Photocurrent dynamics in a poly(phenylene vinylene)-based photorefractive composite, Phys. Rev. B, 69 (2004) 125216.

[23] N.V. Kukhtarev, V.B. Markov, S.G. Odulov, M.S. Soskin, V.L. Vinetskii, holographic storage in electrooptic crystals. II. beam coupling - light amplification, Ferroelectrics, 22 (1978) 961-964.

[24] N. Tsutsumi, W. Miyazaki, Photorefractive performance of polycarbazoylethylacrylate composites with photoconductive plasticizer, J. Appl. Phys., 106 (2009) 083113.

[25] H. Kogelnik, Coupled wave theory for thick hologram gratings, Bell Syst. Tech. J., 48 (1969) 2909-2947.

[26] S. Schloter, U. Hofmann, P. Strohriegl, H.W. Schmidt, D. Haarer, High-performance polysiloxane-based photorefractive polymers with nonlinear optical azo, stilbene, and tolane chromophores, J. Opt. Soc. Am. B, 15 (1998) 2473-2475. 
